\title{
EDITORIAL
}

\section{Posture in the Picture: On the Relevance of Postural Control in Children with Developmental Motor Disorders}

\begin{abstract}
Deficient postural control is a main factor in motor disorders such as cerebral palsy (CP) and developmental coordination disorder (DCD)-disorders which from early childhood seriously interfere with the quality of life. The abnormalities in postural control might be due to deviations in the early development of the CNS or to insults in the pre- and perinatal period, leading to deficiencies in the processing of sensory information and motor output. Recent research has pointed to the considerable compensational capacity of the CNS and possibilities for adaptation at young stages. In the summer of 2004 a workshop was organized in Groningen, the Netherlands in which leading experts on research into sensory systems, motor circuitries and sensori-motor coordination involved in movement and postural control, discussed their recent research findings and their views on motor development. The workshop aimed at covering these fields from neurobiological, neurobehavioral, and clinical perspectives, the latter including the consequences for therapeutic management of children with developmental motor disorders.

In the first section on theoretical issues and postural development, Albert Gramsbergen discusses postural control and extremity movements from an evolutionary perspective and he particularly focuses on the evolution of the motor systems in the CNS. Bert Otten presents a computer model of an articulated threesegment limb, controlled by pairs of muscles which is requested to follow a certain trajectory and he questions whether movements are planned in terms of endpoint equilibrium positions of the limb, or whether the CNS plans movements by selecting appropriate sets of muscle activations with certain durations to move a limb from one point to the other. Mark Latash discusses his Uncontrolled Manifold (UCM) hypothesis, based on the assumption that the CNS organizes covariation of elemental variables to stabilize important performance variables in a task-specific manner. This allows the formulation of testable hypotheses with respect to the development of postural synergies in both typically and atypically developing persons.

Mijna Hadders-Algra reviews the development of postural control during infancy. Two functional levels can be distinguished, a basic level dealing with the generation of direction specific adjustments and a second level, involved in adaptations of the direction specific adjustments. Development probably starts with an innate repertoire of direction specific adjustments. From 6 months onward, the second level of postural control becomes functionally active. Christine Assaiante, reviews earlier studies on the development of postural control during walking, and her message is that children initially build a repertoire of postural strategies, while in a second phase they learn to select the most appropriate strategy in the given circumstances. Her own research showed that anticipatory postural control, despite its relatively early onset only matures slowly during childhood.

In the second session on the development of the motor systems involved in postural control, Laurent Vinay reviews recent literature on brain stem pathways involved in postural control. He particularly focuses on serotonergic pathways in the rat and he extrapolates the results of experimental research to human development. Jos IJkema Paassen reviews literature on the properties of muscles with specific postural tasks as well as the development of their innervation. Most of the data were obtained in animal investigations but she makes attempts to extrapolate these data to the developing human. Jerome Swinny discusses his own research into cerebellar development and that of others, particularly on the development of the two afferent
\end{abstract}


systems to the Purkinje cell which in turn processes and "exports" this information via relay stations to the cerebral cortex and the spinal cord. He particularly stresses the importance of the shift from development towards "plasticity" in the cerebellum and the molecules involved in these processes. Jasper Visser and Bas Bloem discuss the function of the basal ganglia in postural control. Unfortunately, our knowledge on the development of the basal ganglia and on their role in the development of postural control is nil. Visser and Bloem discuss knowledge on processing (or, as they state, the prioritizing of tasks) by the basal ganglia at adult age, hoping thereby that this might give insight into the role of the basal ganglia in the development of postural control.

In the third part particularly dealing with postural control in children, Fallang presents clinical and neurophysiological data of postural control in preterm children without CP. Preterm infants show decreased mobile postural behavior compared with full-term infants. This less mobile postural behavior was related to a better quality of goal-directed reaching during infancy but also to less favorable neuromotor behavior in school-age. Geuze reviews the balance problems of children with Developmental Coordination Disorder (DCD). He concludes that under normal conditions, static balance control is not a problem for children with DCD, but in difficult, unattended or novel situations these children seem to suffer from increased postural sway.

Jolanda van der Heide and Mijna Hadders in their paper give an overview of knowledge currently available on dyscoordination underlying postural problems in children with CP. Obviously, such knowledge is a prerequisite for the development of successful therapeutic interventions in these patients.

Giorgio Porro discuss the ocular and ocular motor disorders which lead to anomalous head control and subsequent abnormal head posture and torticollis in children with developmental motor disorders. Such abnormalities often are overlooked and he points to the role of these abnormalities in an impaired postural control of children with CP. Marjorie Woollacott in her review explores studies related to constraints on balance and walking in children with $\mathrm{CP}$ and the efficacy of training regaining balance after disturbances. The efficiency of keeping balance in such situations can be improved indeed, as indicated by both a reduction in the shifts in the centre of pressure and the time to restabilize balance as a result of training. Eva Brogren Carlberg describes the pathophysiology of postural control in sitting children with $\mathrm{CP}$ and she outlines the requirements and implications for management and treatment. Typically in these children is a top-down recruitment of postural muscles, an excessive degree of co-activation in antagonistic muscles involved in postural maintenance. Therapy should take into account adapted sitting devices and training in motivating settings.

Harris reviews 12 studies (collected after a wide survey) into the effectiveness intervention strategies specifically aiming at improving postural control in children with CP. These studies only limited evidence on improvements and she advocates studies with stronger designs in order to establish that such interventions are effective. Charles and Gordon also discuss recent studies involving intensive rehabilitation in children with hemiplegia and they discuss a number of theoretical considerations. They conclude that substantially more work must be performed before approaches such as constraint-induced therapy and forced-use [this approach] can be advocated for general clinical use.

The papers in this special issue indicate the vivid interest from neurophysiologists, neuroanatomists, and theoreticians into the mechanisms involved in postural control. They demonstrate that our knowledge on these mechanisms has increased impressively over the last years. Also, the knowledge on postural development in the human together with the development of concepts on postural control mechanisms based 
upon principles derived from developmental neurobiology and physical modeling has evolved dramatically. The reports from scientists involved in clinical settings indicate that the moment is approaching that this knowledge and these theoretical insights should be applied in children with CP and DCD and that these studies should be paralleled by rigorous investigations into the efficacy of new therapies. This means that this special issue may be regarded as a significant milestone in our knowledge on postural control.

\section{Albet Gramsbergen,}

Mijna Hadders-Algra

Groningen, the Netherlands 

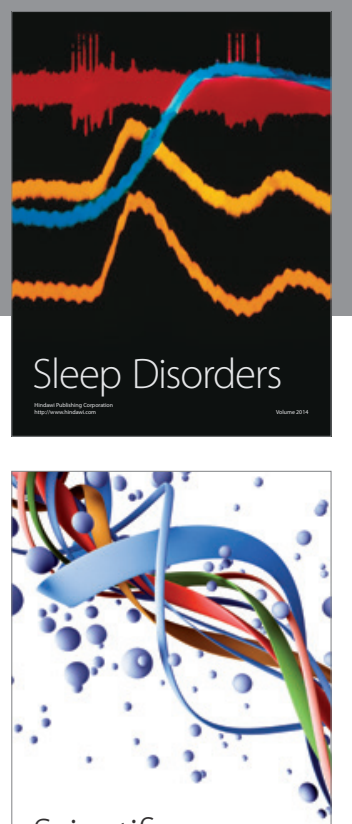

Scientifica
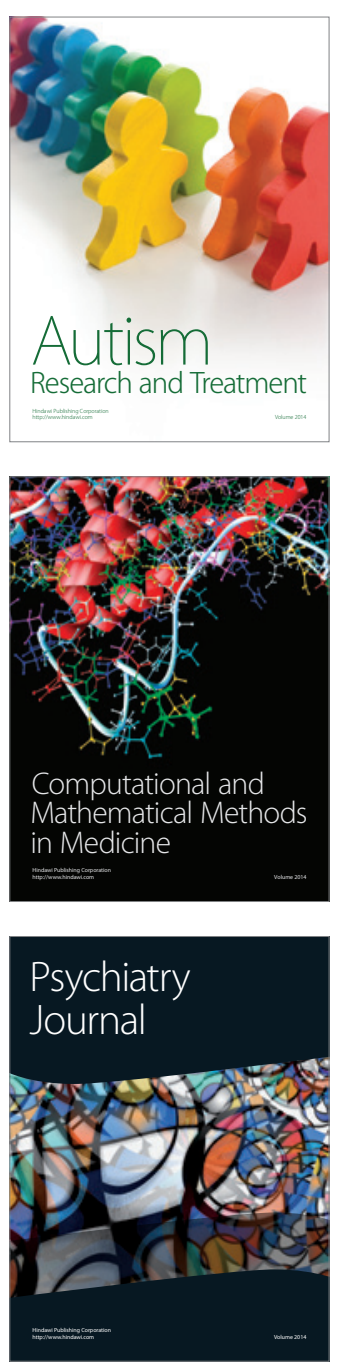
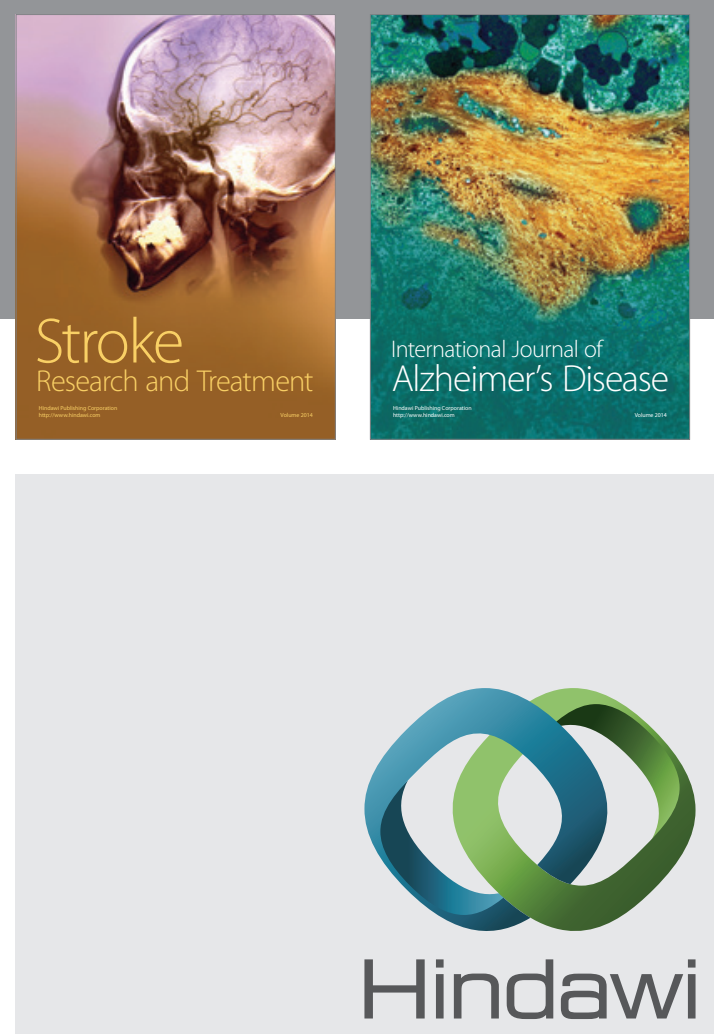

Submit your manuscripts at

http://www.hindawi.com
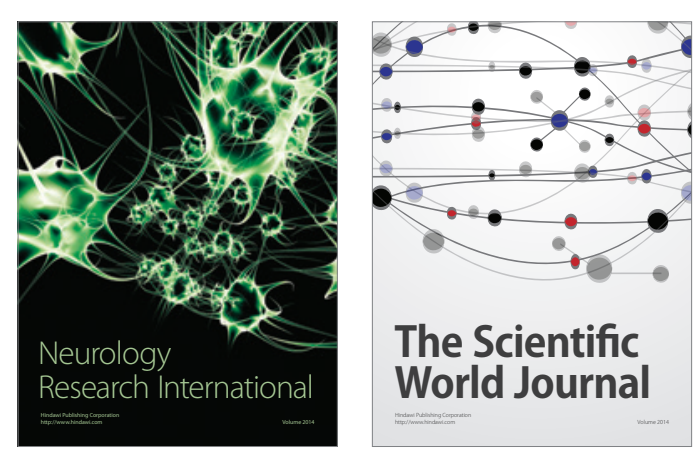

The Scientific World Journal

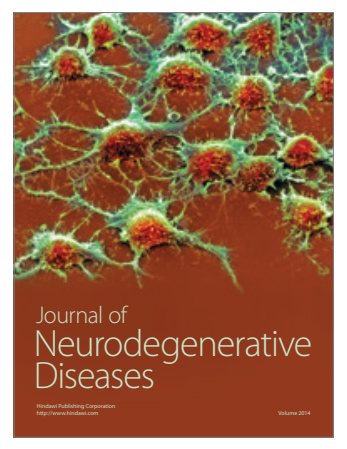

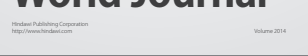

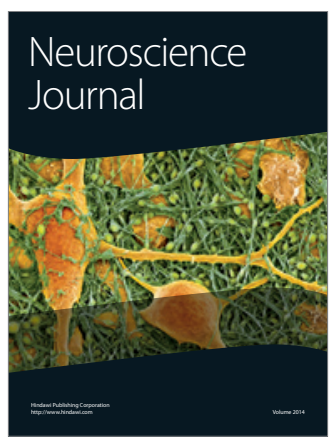

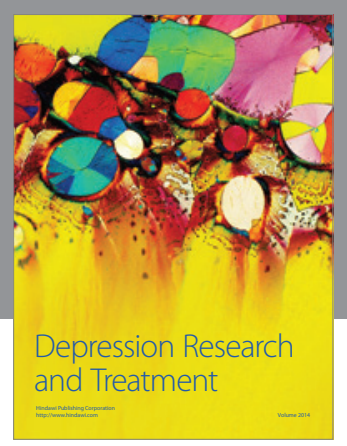
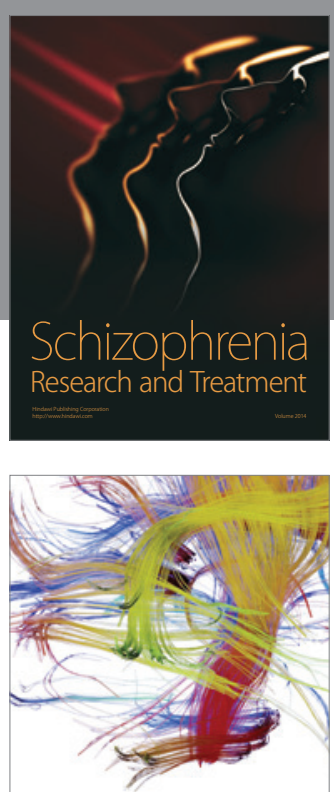

Brain Science

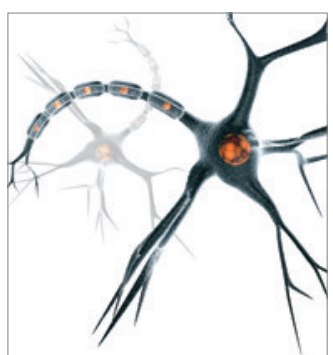

Neural Plasticity
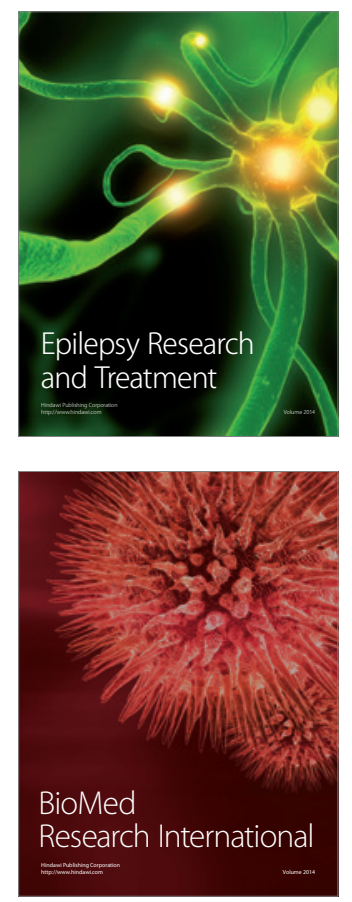

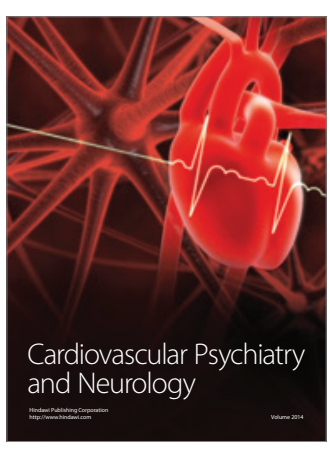

Parkinson's

Disease
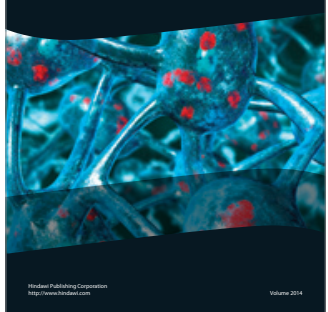